\title{
Generalizations of exactly solvable quantum spin models
}

\author{
A. A. Zvyagin \\ Max-Planck-Institut für Physik komplexer Systeme, 01187 Dresden, Germany; \\ B. I. Verkin Institute for Low Temperature Physics and Engineering of the National Academy of Sciences of Ukraine, Kharkiv 61103, Ukraine; \\ and V. N. Karazin Kharkiv National University, 4 Svoboda Square, Kharkiv 61022, Ukraine
}

(Received 10 October 2019; revised manuscript received 13 November 2019; accepted 21 January 2020; published 2 March 2020)

\begin{abstract}
Several generalizations of one-dimensional and two-dimensional exactly solvable low-dimensional quantum spin-1/2 models, some of which contain off-diagonal terms of the exchange tensor, are proposed. It is shown that off-diagonal terms of the exchange (symmetric or nonsymmetric with respect to the exchange of spins) yield renormalization of the diagonal ones and phase shifts. The latter can be moved to eigenfunctions of the models and do not affect eigenvalues for open geometries of the lattices. For closed geometries of the lattices the phase shifts manifest themselves in the finite size corrections. The advantage of the proposed models is in their simplicity and in possible realizations of the models in a number of applications, e.g., for the description of the wide variety of correlated electron systems, ultracold atoms, and in the theory of topological quantum computation.
\end{abstract}

DOI: 10.1103/PhysRevB.101.094403

\section{INTRODUCTION}

In the first quantum mechanical theories of magnetism [1] the direct exchange interaction was proposed to be the consequence of the Coulomb interaction between electrons carrying spin-1/2. It was proved that namely such an interaction is mainly responsible for the long-range magnetic ordering in condensed-matter physics. The Hamiltonian of the exchange coupling $\mathcal{H}=-J \sum_{n m}\left(\mathbf{S}_{n} \cdot \mathbf{S}_{m}\right)$ [here $\mathbf{S}_{n}$ is the spin operator of the $n$th electron, $(\mathbf{a b})$ is the scalar product, and $J>0$ is determined by the Coulomb integral and the exchange integral] was supposed to be isotropic in spin components and symmetric under the exchange of spins. Then it was recognized [2] that the sign of $J$ can be also negative, depending on the values of the Coulomb integral, the "bare" exchange integral, and the overlap integral. Much later it was proposed to take the relativistic spin-orbit interaction into account [3], within the formalism of the indirect exchange coupling [4]. The overlaps of electron wave functions (the key ingredient of the exchange interaction) appeared to be strongly dependent on positions and orientations of the wave functions of magnetic ions and ligands (nonmagnetic ions, surrounding magnetic ones, via which the indirect exchange/superexchange coupling was realized). The theory of the anisotropic exchange interaction was developed, e.g., in Ref. [5]. The Hamiltonian of the exchange coupling of spins of electrons in the general form can be written as $\mathcal{H}=\sum_{n m} \sum_{\beta, \beta^{\prime}=x, y, z} S_{n}^{\beta} J_{|n-m|}^{\beta, \beta^{\prime}} S_{m}^{\beta^{\prime}}$, where $J_{|n-m|}^{\beta, \beta^{\prime}}$ is the $3 \times 3$ tensor. It means that in general

Published by the American Physical Society under the terms of the Creative Commons Attribution 4.0 International license. Further distribution of this work must maintain attribution to the author(s) and the published article's title, journal citation, and DOI. Open access publication funded by the Max Planck Society. nine independent parameters govern the anisotropy of the exchange interaction. The exchange coupling usually rapidly decays with distance between spins $|n-m|$. In what follows we limit ourselves with the nearest-neighbor spins, and for simplicity we use the notation $J^{\beta \beta^{\prime}}$. The components of the exchange tensor are determined by the spin-orbit interaction of electrons, the symmetry of the positions, and the orientations of the electron wave functions of magnetic ions and ligands in the magnetic crystal. In the absence of the external magnetic field the tensor $J^{\beta \beta^{\prime}}$ can be diagonalized, so that only three main diagonal components $J^{\beta \beta}$ define the magnetic anisotropy. However, when studying the properties of real magnetic materials one often has to consider off-diagonal components. For instance, nonsymmetric under the exchange of spins off-diagonal components of the exchange interaction are known as the Dzyaloshinskii-Moria (DM) coupling [5,6]. Symmetric off-diagonal components (sometimes called the $\Gamma$ couplings) appear to be very important in the interaction of orbitals of strongly correlated electron systems [7], in particular in the so-called compass models [8] or in the socalled Kitaev materials [9], where their origin is related to pseudodipolar interactions, and also in Rydberg cold atoms with van der Waals interactions [10] and optical lattices with trapped ultracold fermions [11].

Unlike higher-dimensional models, their low-dimensional quantum counterparts permit one to obtain exact (nonperturbative) results [12]. The necessity of nonperturbative studies in low-dimensional quantum systems is caused by the enhanced quantum and thermal fluctuations there, due to the features in the density of states [13]. Exactly solvable models are not only important for the pure quantum many-body theory. Nowadays they are extremely useful in a number of areas, which are described by that theory, like real quasi-low-dimensional spin systems, manufactured during the last decade, topological insulators, ultracold atoms in optical traps, strongly correlated electrons interacting via 
superexchange orbital interactions, sets of qubits (subjects of quantum information and computation), etc. Most of the mentioned issues cannot be explained in the framework of weak couplings (the perturbative theory) or the mean-field-like approach. Low-dimensional exact quantum solutions provide a complete and unambiguous picture of correlated quantum many-body systems and play an essential role as the basis for further applications of perturbative and mean-field-like methods. Low-dimensional quantum spin models often reveal properties of quantum spin liquids. In the latter the magnetic order is suppressed down to the lowest temperatures due to the frustration of spin-spin interactions and/or enhanced quantum fluctuations in low-dimensional systems [14]. In many quantum spin liquids, emergent magnetic excitations are fermions (as a rule they carry fractionalized spin), instead of magnons (bosons, which carry spin 1), for ordered magnetic systems. Notice that, while bosons have their classical counterparts, fermions, instead, have a totally quantum nature.

In this paper, we consider several generalizations of the well-known spin- $1 / 2$ exactly solvable models. Our goal is to find other exactly solvable quantum spin models, in particular, the ones with off-diagonal components of the exchange tensor.

\section{CONSTRUCTION OF ONE-DIMENSIONAL EXACTLY SOLVABLE MODELS}

The idea of the present work can be shown using the known example: The one-dimensional spin- $1 / 2$ chain with the uniaxial magnetic anisotropy, which contains off-diagonal terms of the exchange tensor, namely, the DM interaction. The Hamiltonian has the form $\mathcal{H}_{D}=\sum_{n}\left[J\left(S_{n}^{x} S_{n+1}^{x}+\right.\right.$ $\left.\left.S_{n}^{y} S_{n+1}^{y}\right)+D\left(S_{n}^{x} S_{n+1}^{y}-S_{n}^{y} S_{n+1}^{x}\right)+J_{z} S_{n}^{z} S_{n+1}^{z}\right]$. Here $S_{n}^{x, y, z}$ are the operators of the projections of the spin-1/2 situated at the site $n ; J$ and $J_{z}$ are the components of the anisotropic (uniaxial) exchange interaction; and $D$ is the DM coupling. The model is known to be exactly solvable [15] The simplest way to prove its integrability is to rewrite it in the form $\mathcal{H}_{D}=(1 / 2) \sum_{n}\left[\sqrt{J^{2}+D^{2}}\left(S_{n}^{+} S_{n+1}^{-} e^{i \phi}+\right.\right.$ H.c. $)+$ $2 J_{z} S_{n}^{z} S_{n+1}^{z}$, with the cylic components $S_{n}^{ \pm}=S_{n}^{x} \pm i S_{n}^{y}$ and $\tan \phi=D / J$. Then we use the unitary transformation $\mathcal{H}_{1}=$ $U^{-1} \mathcal{H} U$, with $U=\exp \left[-i(\phi) \sum_{j} j S_{j}^{z}\right]$. For the open chain, that transformation removes the phase factor $\phi$, related to the DM interaction, from the Hamiltonian (for the ring the latter is translated to the twisted boundary conditions, which are realized within the Bethe ansatz equations for quasimomenta [15] and reveal themselves in the finite size corrections [16]). Hence, using the unitary transformation, the system with the Hamiltonian, which contains nonzero off-diagonal components of the exchange tensor, is exactly mapped onto the exactly solvable Hamiltonian of the XXZ chain with renormalized values of the diagonal (transverse, $x x$ and $y y$ ) components of the exchange tensor. Obviously, the unitary transformation does not change the eigenvalues of the Hamiltonian (up to mentioned renormalization of the diagonal components, naturally). However, the phase factor, which is removed from the Hamiltonian, is present in the set of eigenfunctions. As such, it manifests itself in average values of many operators. In particular, the phase factor, related to the DM coupling, reveals itself in the nonzero components of correlation functions like $\left\langle S_{n}^{x} S_{m}^{y}\right\rangle$. For example, the ground- state correlation function $\left\langle S_{n}^{x} S_{n+r}^{y}-S_{n}^{y} S_{n+r}^{x}\right\rangle$ for the critical chain $-\sqrt{J^{2}+D^{2}} \leqslant J_{z} \leqslant \sqrt{J^{2}+D^{2}}$ for $r=1$ is equal to

$$
\left\langle S_{n}^{x} S_{n+1}^{y}-S_{n}^{y} S_{n+1}^{x}\right\rangle=\frac{D}{4 f_{1}}\left[I_{1}+\frac{J_{z}}{\sqrt{J^{2}+D^{2}}} I_{2}\right],
$$

where $f_{1}=\sqrt{J^{2}+D^{2}-J_{z}^{2}}$,

$$
\begin{aligned}
I_{1}= & \int_{-\infty}^{\infty} \frac{d x \sinh (\lambda x)}{\sinh (\pi x) \cosh [(\pi-\lambda) x]}, \\
I_{2}= & \int_{-\infty}^{\infty} \frac{x d x}{\sinh (\pi x) \cosh [(\pi-\lambda) x]} \\
& \times\left[\cosh (\lambda x)+\frac{\sinh (\lambda x) \sinh [(\pi-\lambda) x]}{\cosh [(\pi-\lambda) x]}\right],
\end{aligned}
$$

where $\cos \lambda=-J_{z} / \sqrt{J^{2}+D^{2}}$. On the other hand, for $J_{z}>$ $\sqrt{J^{2}+D^{2}}$, the ground-state correlation function is

$$
\left\langle S_{n}^{x} S_{n+1}^{y}-S_{n}^{y} S_{n+1}^{x}\right\rangle=\frac{D}{2 f_{2}}\left[-S_{1}+\frac{f_{2} J_{z}}{\left(J^{2}+D^{2}\right)} S_{2}\right],
$$

where $f_{2}=\sqrt{J_{z}^{2}-J^{2}-D^{2}}$,

$$
\begin{aligned}
& S_{1}=\sum_{m=-\infty}^{\infty} e^{-2 n v} \tanh (n v), \\
& S_{2}=\sum_{m=-\infty}^{\infty} n e^{-2 n v}\left[\left(1-\tanh ^{2}(n v)-2 \tanh (n v)\right],\right.
\end{aligned}
$$

with $\cosh v=-J_{z} / \sqrt{J^{2}+D^{2}}$. For the ferromagnetic case $J_{z}<-\sqrt{J^{2}+D^{2}}$, the correlation function is zero. Obviously for the zero nondiagonal components of the exchange tensor, $D=0$, such a correlation function is zero (as well as similar correlation functions for any $r$ ). Those correlation functions determine the spiral structure of the transverse components with the spiral (pitch) angle $\phi$ in the system, caused by the DM interaction [15]. Then it is not difficult to generalize such a scheme for the construction of the exactly solvable Hamiltonian of the dimerized spin-1/2 chain with the DM interaction [17].

To follow our strategy, we consider then the spin- $1 / 2$ chain with the biaxial magnetic anisotropy and with off-diagonal exchange couplings of the DM type:

$$
\begin{gathered}
\mathcal{H}_{b D}=\sum_{n}\left[J_{x} S_{n}^{x} S_{n+1}^{x}+J_{y} S_{n}^{y} S_{n+1}^{y}+D\left(S_{n}^{x} S_{n+1}^{y}\right.\right. \\
\left.\left.-[J y / J x] S_{n}^{y} S_{n+1}^{x}\right)+J_{z} S_{n}^{z} S_{n+1}^{z}\right] .
\end{gathered}
$$

Let us rotate each second spin around the axis $z$ through the angle $\phi=\tan ^{-1}\left[-D / J_{x}\right]$. Then the phase factor can be removed from the Hamiltonian (or translated to twisted boundary condition) using that unitary transformation. We see that the same properties are pertinent to that new exactly solvable model: The Hamiltonian of the system with nonsymmetric off-diagonal components of the exchange tensor is exactly mapped to the exactly solvable Hamiltonian of the XYZ spin$1 / 2$ chain with the renormalized diagonal components of the exchange tensor. The phase factor defines the pitch angle of the spiral structure in the $x y$ spin space, which exists due to nonzero values of correlation functions like $\left\langle S_{n}^{x} S_{m}^{y}\right\rangle$, etc. [15]. 
It turns out that the unitary transformations, which we used above, are the nonhomogeneous rotations of spins of the chain around the $z$ axis. Namely, because of that reason the unitary transformation does not affect the $z$ components of spins, and, hence, $z z$ components of the exchange tensor. Hence, such and similar unitary transformations can be used for the models with the external magnetic field, applied along the $z$ axis.

Now we can use another transformation to show that the spin-1/2 chain Hamiltonian with symmetric off-diagonal components of the exchange tensor is also exactly solvable. Consider the Hamiltonian

$$
\begin{aligned}
\mathcal{H}_{b 2}=\sum_{n}( & A\left\{\left(J_{x}[1-a]+J_{y} a\right) S_{n}^{x} S_{n+1}^{x}+\left(J_{y}[1-a]\right.\right. \\
& \left.+J_{x} a\right) S_{n}^{y} S_{n+1}^{y}+\sqrt{a(1-a)}\left(J_{y}-J_{x}\right)\left(S_{n}^{x} S_{n+1}^{y}\right. \\
& \left.\left.\left.+S_{n}^{y} S_{n+1}^{x}\right)\right\}+J_{z} S_{n}^{z} S_{n+1}^{z}\right),
\end{aligned}
$$

with $0 \leqslant a \leqslant 1$. The case $a=0$ describes the standard XYZ chain, and the case $a=1$ interchanges the values of the diagonal transverse components of the exchange tensor. The Hamiltonian can be rewritten as

$$
\begin{gathered}
\mathcal{H}_{b 2}=\frac{1}{4} \sum_{n}\left\{A\left[J_{+} S_{n}^{+} S_{n+1}^{-}+A J_{-}\left(S_{n}^{+} S_{n+1}^{+} e^{2 i \theta}\right)+\text { H.c. }\right]\right. \\
\left.+4 J_{z} S_{n}^{z} S_{n+1}^{z}\right\},
\end{gathered}
$$

where $S_{n}^{ \pm}=S_{n}^{x} \pm i S_{n}^{y}$ are the operators of the cyclic projections of spins, $J_{ \pm}=\left(J_{x} \pm J_{y}\right) / 2$, and $\theta=\sin ^{-1} \sqrt{a}$. That Hamiltonian is the one of the XYZ chain after the homogeneous rotation of spins around the $z$ axis through the angle $\theta$. To prove the exact integrability of the Hamiltonian, we can first use the Jordan-Wigner transformation [18]: $\quad S_{n}^{z}=(1 / 2)\left(1-2 a_{n}^{\dagger} a_{n}\right), \quad S_{n}^{+}=\prod_{m=0}^{n-1}\left(1-2 a_{m}^{\dagger} a_{m}\right) a_{n}$, and $S_{n}^{-}=a_{n}^{\dagger} \prod_{m=0}^{n-1}\left(1-2 a_{m}^{\dagger} a_{m}\right)$, where $a_{n}^{\dagger} \quad\left(a_{n}\right)$ is the Dirac operator of creation (destruction) of the fermion in the site $n$. The Hamiltonian gets the form $\mathcal{H}_{b 2}=-(1 / 4) \sum_{n}\left\{A\left[J_{+}\left(a_{n}^{\dagger} a_{n+1}\right)+J_{-} a_{n}^{\dagger} a_{n+1}^{\dagger} e^{-2 i \theta}+\right.\right.$ H.c. $]+$ $\left.J_{z}\left(1-2 a_{n}^{\dagger} a_{n}\right)\left(1-2 a_{n+1}^{\dagger} a_{n+1}\right)\right\}$. Then, using the unitary transformation $a_{n}^{\dagger}=e^{i \theta} \tilde{a}_{n}^{\dagger}$, with $a_{n}=e^{-i \theta} \tilde{a}_{n}$, and using the inverse of the Jordan-Wigner transformation, we can finally obtain the Hamiltonian in the form of the one of the XYZ spin-1/2 chain, in which diagonal $x x$ and yy coefficients of the exchange tensor are multiplied by $A$. Removing the phase $\theta$ from the Hamiltonian we get eigenvalues independent of it, while eigenfunctions do depend on it. At $J_{z}=0$, for $J_{x}>J_{y}$ the model is ordered in the ground state [19] with the components of the order parameters $m^{x} \equiv \sqrt{\lim _{m \rightarrow \infty}(-1)^{m}\left\langle S_{n}^{x} S_{n+m}^{x}\right\rangle}$ and $m^{y} \equiv \sqrt{\lim _{m \rightarrow \infty}(-1)^{m}\left\langle S_{n}^{y} S_{n+m}^{y}\right\rangle}$ equal to

$$
\begin{aligned}
& m^{x}=\sqrt{\frac{J_{+}}{2 J_{x}}}\left(\frac{J_{-}}{J_{+}} \cos ^{2} \theta\right)^{1 / 4}, \\
& m^{y}=\sqrt{\frac{J_{+}}{2 J_{x}}}\left(\frac{J_{-}}{J_{+}} \sin ^{2} \theta\right)^{1 / 4} ;
\end{aligned}
$$

i.e., the ordering is tilted. The phase factor $\theta$ also produces nonzero nondiagonal correlation functions like $\left\langle S_{n}^{x} S_{n+m}^{y}\right\rangle$. For example, we have

$$
\lim _{m \rightarrow \infty}(-1)^{m}\left\{\left(S_{n}^{x} S_{n+m}^{y}+S_{n}^{y} S_{n+m}^{x}\right)\right\rangle=\frac{J_{+}}{2 J_{x}}\left(\frac{J_{-}^{2}}{J_{+}^{2}} \cos \theta \sin \theta\right)^{1 / 4} .
$$

If $J_{y}>J_{x}$, then one has to interchange $J_{x} \leftrightarrow J_{y}$ and $\cos ^{2} \theta \leftrightarrow$ $\sin ^{2} \theta$. If the external magnetic field $h$ along the $z$ axis is switched on, the components of the order parameter and the nondiagonal correlation function disappear as $\left[1-\left(h / J_{+}\right)\right]^{1 / 8}$ and $\left[1-\left(h / J_{+}\right)\right]^{1 / 4}$, respectively. The action of the phase $\theta$ is equivalent to the action of the phase of the order parameter in the superconductor; hence, it can reveal itself, e.g., in the Josephson-like coupling between chains [20], in the circuit QED one-dimensional system of superconducting charged qubits [21], and in the braiding of topologically protected edge Majorana modes belonging to coupled chains [22] for $J_{z}=0$.

Consider the dimerized spin-1/2 chain model with the Hamiltonian containing symmetric off-diagonal components of the exchange tensor:

$$
\begin{aligned}
\mathcal{H}_{b d}= & -\sum_{n}\left[A \left\{\left(J_{1 x}[1-a]+J_{1 y} a\right) S_{n-1}^{x} S_{n}^{x}+\left(J_{1 x} a+[1-a] J_{1 y}\right) S_{n-1}^{y} S_{n}^{y}+\left(J_{1 y}-J_{1 x}\right) \sqrt{a(1-a)}\left(S_{n-1}^{x} S_{n}^{y}+S_{n-1}^{y} S_{n}^{x}\right)\right.\right. \\
& \left.+\left(J_{2 x}[1-a]+J_{2 y} a\right) S_{n}^{x} S_{n+1}^{x}+\left(J_{2 x} a+[1-a] J_{2 y}\right) S_{n}^{y} S_{n+1}^{y}+\left(J_{2 y}-J_{2 x}\right) \sqrt{a(1-a)}\left(S_{n}^{x} S_{n+1}^{y}+S_{n}^{y} S_{n+1}^{x}\right)\right\} \\
& \left.+H\left(\mu_{1} S_{n-1}^{z}+\mu_{2} S_{n}^{z}\right)\right],
\end{aligned}
$$

with $0 \leqslant a \leqslant 1$. Here $H$ is the external magnetic field along the axis $z$, and $\mu_{1,2}$ are the effective magnetons for the even and odd sites of the chain. (Notice that $z z$ coupling can be added; however, the obtained Hamiltonian is not exactly solvable.) The limiting case $J_{2 x}=J_{1 y}=0$ describes the onedimensional compass model or the one-dimensional limiting case of the Kitaev honeycomb model. We can apply the Jordan-Wigner transformation, and the consequent transformation to Majorana fermions, which removes the phase shift $\theta=\sin ^{-1} \sqrt{a}$ from the Hamiltonian. Then we diagonalize the obtained Hamiltonian. The eigenvalues of the Hamiltonian coincide with ones of the known exactly solvable dimerized spin- $1 / 2$ chain model with only diagonal components of the exchange tensor [23].

The average values of the sum and the difference of the $z$ projections of the two neighboring spins are

$$
\left\langle S_{n-1}^{z} \pm s_{n+1}^{z}\right\rangle=\frac{\left(\mu_{1} \pm \mu_{2}\right) H}{2 N} \sum_{k} \frac{\sqrt{\alpha_{k}^{2}+\left|\beta_{k}\right|^{2}} \pm \alpha_{k}}{\varepsilon_{k 1} \varepsilon_{k 2}\left(\varepsilon_{k 1}+\varepsilon_{k 2}\right)}
$$

where $\quad \alpha_{k}=\mu_{1} \mu_{2} H^{2}-\left(A^{2} / 4\right)\left[J_{1 x} J_{1 y}+J_{2 x} J_{2 y}+\left(J_{1 x} J_{2 x}+\right.\right.$ $\left.\left.J_{1 y} J_{2 y}\right) \cos (2 k)\right]$ and $\beta_{k}=-\left(i A^{2} / 4\right) \sin (2 k)\left(J_{1 x} J_{2 y}-J_{1 y} J_{2 x}\right)$. Depending on the values of $H, \mu_{12}, J_{1,2, x, y}$, and $A$, there 
can be two, one, or no quantum phase transitions in the model [23]. It is easy to show that there exist two zero energy edge Majorana modes in the open chain model for $H=0$. The braiding between such topologically protected zero modes can manifest itself in operations with qubits in one-dimensional topological quantum computers. The removed from the Hamiltonian phase is again present in the eigenfunctions and can be seen, e.g., in transverse correlation functions.

\section{CONSTRUCTION OF TWO-DIMENSIONAL EXACTLY SOLVABLE MODELS}

Finally, let us consider two exactly solvable generalizations of the two-dimensional model, namely, the Kitaev honeycomb spin-1/2 model [24] with off-diagonal components of the exchange tensor. For that purpose it is convenient to use the equivalence of the honeycomb and the brick-wall lattices and the generalization of the Jordan-Wigner transformation [25]. The Hamiltonian of the first model reads

$$
\begin{aligned}
\mathcal{H}_{K 1}= & \sum_{j+n=\text { even }}\left[J_{1} S_{j, n}^{x} S_{j+1, n}^{x}+J_{2} S_{j-1, n}^{y} S_{j, n}^{y}\right. \\
& \left.+D\left(S_{j, n}^{x} S_{j+1, n}^{y}-\left[J_{2} / J_{1}\right] S_{j-1, n}^{y} S_{j, n}^{x}\right)+J_{3} S_{j, n}^{z} S_{j, n+1}^{z}\right]
\end{aligned}
$$

i.e., it contains nonsymmetric (DM-type) off-diagonal components of the exchange tensor. Using the rotation of each $j+n=$ odd spin about the $z$ axis through the phase $\phi=$ $\tan ^{-1}\left[-D / J_{1}\right]$, we get the standard Kitaev model with the $\phi$ dependence either totally removed from the Hamiltonian (for the open geometry in the respective direction of the brick-wall lattice) or present in the twist boundary conditions for the closed geometry. For the latter, it manifests itself in the finite size corrections to the energy. The phase factor defines the behavior of the transverse correlation functions, producing the spiral structure with the angle $\phi$ along the $x$ axis of the brick-wall lattice.

The Hamiltonian of the second exactly solvable Kitaev-like model with off-diagonal components of the exchange tensor has the form

$$
\begin{aligned}
\mathcal{H}_{K 2}= & \sum_{j+n=\text { even }}\left\{A \left[J_{1}(1-a) S_{j, n}^{x} S_{j+1, n}^{x}+J_{1} a S_{j, n}^{y} S_{j+1, n}^{y}\right.\right. \\
& -J_{1} \sqrt{a(1-a)}\left(S_{j, n}^{x} S_{j+1, n}^{y}+S_{j, n}^{y} S_{j+1, n}^{x}\right) \\
& +J_{2} a S_{j-1, n}^{x} S_{j, n}^{x}+J_{2}(1-a) S_{j-1, n}^{y} S_{j, n}^{y}+J_{2} \sqrt{a(1-a)} \\
& \left.\left.\times\left(S_{j-1, n}^{x} S_{j, n}^{y}+S_{j-1, n}^{y} S_{j, n}^{x}\right)\right]+J_{3} S_{j, n}^{z} S_{j, n+1}^{z}\right\}
\end{aligned}
$$

with $0 \leqslant a \leqslant 1$; i.e., it contains symmetric off-diagonal components of the exchange tensor. We can use the generalization of the Jordan-Wigner transformation [25]:

$$
\begin{aligned}
& S_{j, n}^{+}=\exp \left(i \pi\left[\sum_{l, m<n} a_{l, m}^{\dagger} a_{l, m}+\sum_{l<j} a_{l, n}^{\dagger} a_{l, n}\right]\right) a_{j, n}, \\
& S_{j, n}^{-}=\left(S_{j, n}^{+}\right)^{+}, \quad S_{j, n}^{z}=\frac{1}{2}-a_{j, n}^{\dagger} a_{j, n},
\end{aligned}
$$

with Dirac fermion operators $a_{j, n}^{\dagger}$ and $a_{j, n}$. Then for the even $j+n$, we can use the transformations $a_{j, n}^{\dagger}=e^{-i \theta}\left(d_{j, n}-i c_{j, n}\right)$ and $a_{j, n}=e^{i \theta}\left(d_{j, n}+i c_{j, n}\right)$, with $c_{j, n}$ and $d_{j, n}$ being the Majorana fermion operators and $\theta=\sin ^{-1} \sqrt{a}$. For the odd $j+$ $n$, we apply the transformations $a_{j, n}^{\dagger}=e^{-i \theta}\left(c_{j, n}-i d_{j, n}\right)$ and $a_{j, n}=e^{i \theta}\left(c_{j, n}-i d_{j, n}\right)$. Then the Hamiltonian can be written as

$$
\begin{gathered}
\mathcal{H}_{K 2}=-\frac{i}{4} \sum_{j+n=\mathrm{even}}\left(A J_{1} c_{j, n} c_{j+1, n}-A J_{2} c_{j-1, n} c_{j, n}\right. \\
\left.+4 J_{3} D_{j n} c_{j, n} c_{j, n+1}\right)
\end{gathered}
$$

with the quantum numbers $D_{j n}=i d_{j, n} d_{j, n+1}$ defined on each vertical bond of the brick-wall lattice. Those numbers commute mutually and with the Hamiltonian; hence they act as the static $Z_{2}$ field. Obviously, the Majorana representation of the Hamiltonian (13) (with off-diagonal components of the exchange tensor) coincides up to the renormalization multiplier $A$ with the Majorana representation of the Kitaev model with only diagonal components of the exchange tensor. Again, the eigenvalues of $\mathcal{H}_{K 2}$ do not depend on the removed phase $\theta$, while eigenfunctions do depend on that phase. The dependence on $\theta$ can be manifested in the behavior of the transverse correlation functions, in particular in nonzero values of $\left\langle S_{n}^{x} S_{m}^{y}\right\rangle$, etc. For the open chain and open ladder limits of the model Majorana, zero modes exist. Those modes can manifest themselves in, e.g., the behavior of the topological Pontryagin index (the winding number) in the momentum space [26].

It is interesting to point out the limiting case of Eq. (13). For $A=2, a=1 / 2$, and $J_{1}=J_{2}$, we get the two-dimensional exactly solvable model with the Hamiltonian

$$
\mathcal{H}_{K 2}=\sum_{j, n}\left(J_{1}\left[S_{j, n}^{x} S_{j+1, n}^{x}+S_{j, n}^{y} S_{j+1, n}^{y}\right]+J_{3} S_{j, n}^{z} S_{j, n+1}^{z}\right) .
$$

That brick-wall model manifests the absence of the anisotropy between $x$ and $y$ axes in the spin space. Eigenvalues are the same as those in the standard Kitaev model for $J_{1}=J_{2}$. The model on the honeycomb lattice is also exactly solvable and has similar properties.

\section{ANALYSIS}

Most of the proposed exactly solvable models manifest the hidden nonlocal string order parameters [25,27].

It is possible to construct exactly solvable models combining symmetric and nonsymmetric off-diagonal components of the exchange tensor. The solution can be obtained by the combination of several unitary transformations, which move the phases from the eigenvalues of the Hamiltonian to the eigenfunctions. Similar generalizations can be obtained for the exactly solvable models with three-spin interactions, for example, for one-dimensional and two-dimensional models, containing terms like $S_{n-1}^{x, y} S_{n}^{z} S_{n+1}^{x, y}$, etc. For such models it is also possible to introduce either DM-like nonsymmetric off-diagonal terms or symmetric ones, using the procedure described above. It is also possible to generalize other exactly solvable spin-1/2 models [28] (as well as higher-spin 
models and models on higher-dimensional lattices) by adding symmetric and/or nonsymmetric components of the exchange tensor, as is proposed in the present study.

The phases, which appear in the proposed generalizations of exactly solvable Hamiltonians, for systems like [7-11] have the internal spin-orbit nature. However, one can consider such phases as the consequence of the external magnetic or electric fluxes piercing closed periodic lattices (as one-dimensional rings or two-dimensional cylinders). Such phases (mostly related to the nonsymmetric off-diagonal case) yield the Aharonov-Bohm-Casher effect [29] and produce persistent currents in closed geometries [30]. Similar phases, caused by the external magnetic fluxes, in the symmetric off-diagonal case were recently proposed to be present in Mott insulators with spin-orbit coupling [31].

\section{SUMMARY}

In summary, we have proposed several generalizations of one-dimensional and two-dimensional exactly solvable quan- tum spin- $1 / 2$ models, some of which contain nonsymmetric or symmetric, with respect to the exchange of spins, off-diagonal terms of the exchange tensor. Off-diagonal terms produce renormalization of the diagonal ones, and phase shifts, which can be moved to the eigenfunctions of the system, and do not affect eigenvalues for open lattices, or manifest themselves in finite size corrections for closed geometries of the lattices. The advantage of the proposed models in comparison to other exactly solvable models is in their simplicity and in possible realizations of the models in a number of applications. For example, proposed exactly solvable models can be used in the theoretical description of correlated electron systems with orbital exchange couplings, quantum spin liquids, and ultracold fermionic atoms trapped in optical lattices, and in the models of topological quantum computers using fault-tolerant anyonic computation.

\section{ACKNOWLEDGMENT}

The support from Deutsche Forschungsgemeinschaft (DFG) via Grant No. SFB 1143 is acknowledged.
[1] W. Heisenberg, Z. Phys. 38, 411 (1926); P. A. M. Dirac, Proc. R. Soc. London, Ser. A 112, 661 (1926); W. Heisenberg, Z. Phys. 49, 619 (1928); P. A. M. Dirac, Proc. R. Soc. London, Ser. A 123, 714 (1929); J. H. Van Vleck, Phys. Rev. 45, 405 (1934); J. Frenkel, Z. Phys. 49, 31 (1928); J. Frenkel and J. Dorfman, Nature (London) 126, 274 (1930).

[2] W. Heitler, Z. Phys. 46, 47 (1927); 47, 835 (1928); 51, 805 (1928); F. London, ibid. 46, 455 (1928); 50, 24 (1928).

[3] J. H. Van Vleck, J. Phys. Radium 12, 262 (1951); T. Moriya and K. Yosida, Prog. Theor. Phys. 9, 663 (1953).

[4] H. Kramers, Physica 1, 182 (1934); P. W. Anderson, Phys. Rev. 79, 350 (1950); 115, 2 (1959); J. B. Goodenough, Magnetism and the Chemical Bond (Interscience, New York, 1963).

[5] T. Moriya, Phys. Rev. Lett. 4, 228 (1960); Phys. Rev. 120, 91 (1960).

[6] I. E. Dzyaloshinskii, Zh. Eksp. Teor. Fiz. 32, 1547 (1957) [Sov. Phys. JETP 5, 1259 (1958)]; J. Phys. Chem. Solids 4, 241 (1958).

[7] K. I. Kugel and D. I. Khomskii, Sov. Phys. Usp. 25, 231 (1982); P. Horsch, J. Jaklic, and F. Mack, Phys. Rev. B 59, 6217 (1999); J. van den Brink, P. Horsch, F. Mack, and A. M. Oleś, ibid. 59, 6795 (1999); A. M. Oleś, G. Khaliullin, P. Horsch, and L. F. Feiner, ibid. 72, 214431 (2005); M. Biskup, Z. Nussinov, and L. Chayes, Commun. Math. Phys. 255, 253 (2005); E. Zhao and W. V. Liu, Phys. Rev. Lett. 100, 160403 (2008); C. Wu, ibid. 100, 200406 (2008).

[8] For a recent review, see, e.g., Z. Nussinov and J. van den Brink, Rev. Mod. Phys. 87, 1 (2015).

[9] J. G. Rau, Eric Kin-Ho Lee, and H. Y. Kee, Phys. Rev. Lett. 112, 077204 (2014); R. Yadav, N. A. Bogdanov, V. M. Katukuri, S. Nishimoto, J. van den Brink, and L. Hozoi, Sci. Rep. 6, 37925 (2016); K. Ran, J. Wang, W. Wang, Z.-Y. Dong, X. Ren, S. Bao, S. Li, Z. Ma, Y. Gan, Y. Zhang, J. T. Park, G. Deng, S. Danilkin,
S.-L. Yu, J.-X. Li, and J. Wen, Phys. Rev. Lett. 118, 107203 (2017); L. Janssen, E. C. Andrade, and M. Vojta, Phys. Rev. B 96, 064430 (2017); I. Rousochatzakis and N. B. Perkins, Phys. Rev. Lett. 118, 147204 (2017); S. M. Winter, A. A. Tsirlin, M. Daghofer, J. van den Brink, Y. Singh, P. Gegenwart, and R. Valenti, J. Phys.: Condens. Matter 29, 493002 (2017); P. Lampen-Kelley, S. Rachel, J. Reuther, J.-Q. Yan, A. Banerjee, C. A. Bridges, H. B. Cao, S. E. Nagler, and D. Mandrus, Phys. Rev. B 98, 100403(R) (2018); I. O. Ozel, C. A. Belvin, E. Baldini, I. Kimchi, S. Do, K.-Y. Choi, and N. Gedik, ibid. 100, 085108 (2019); P. Saha, Z. Fan, D. Zhang, and G.-W. Chern, Phys. Rev. Lett. 122, 257204 (2019).

[10] F. Yang, S. Yang, and L. You, Phys. Rev. Lett. 123, 063001 (2019).

[11] J. Simon, W. S. Bakr, R. Ma, M. E. Tai, P. M. Preiss, and M. Greiner, Nature (London) 472, 307 (2011).

[12] See, e.g., A. A. Zvyagin, Quantum Theory of One-Dimensional Spin Systems (Cambridge University Press, Cambridge, England, 2010).

[13] N. D. Mermin and H. Wagner, Phys. Rev. Lett. 17, 1133 (1966).

[14] P. A. Lee, Science 321, 1306 (2008); L. Savary and L. Balents, Rep. Prog. Phys. 80, 016502 (2017).

[15] A. A. Zvyagin, Fiz. Nizk. Temp. 15, 977 (1989) [Fiz. Nizk. Temp. 15, 540 (1989)]; F. C. Alcaraz and W. F. Wreszinski, J. Stat. Phys. 58, 45 (1990); A. A. Zvyagin, J. Phys.: Condens. Matter 3, 3865 (1991).

[16] A. A. Zvyagin, Finite Size Effects in Correlated Electron Systems: Exact results (Imperial College Press, London, 2005).

[17] A. A. Zvyagin, Zh. Eksp. Teor. Fiz. 98, 1396 (1990) [Sov. Phys. JETP 71, 779 (1990)].

[18] P. Jordan and E. Wigner, Z. Phys. 47, 631 (1928).

[19] E. Barough and B. M. McCoy, Phys. Rev. A 3, 786 (1971).

[20] See, e.g., M. Tinkham, Introduction to Superconductivity (McGraw-Hill, New York, 1995). 
[21] W.-L. You, Y. Wang, T.-C. Yi, C. Zhang, and A. M. Oleś, Phys. Rev. B 97, 224420 (2018).

[22] C. Nayak, S. H. Simon, A. Stern, M. Freedman, and S. Das Sarma, Rev. Mod. Phys. 80, 1083 (2008); P. Bonderson, M. Freedman, and C. Nayak, Phys. Rev. Lett. 101, 010501 (2008); A. R. Akhmerov, Phys. Rev. B 82, 020509(R) (2010); J. Alicea, Y. Oreg, G. Refael, F. von Oppen, and M. P. A. Fisher, Nat. Phys. 7, 412 (2011); S. Hoffman, C. Schrade, J. Klinovaja, and D. Loss, Phys. Rev. B 94, 045316 (2016); D. Aasen, M. Hell, R. V. Mishmash, A. Higginbotham, J. Danon, M. Leijnse, T. S. Jespersen, J. A. Folk, C. M. Marcus, K. Flensberg, and J. Alicea, Phys. Rev. X 6, 031016 (2016).

[23] J. H. H. Perk, H. W. Capel, M. J. Zuilhof, and T. J. Siskens, Phys. A (Amsterdam, Neth.) 81, 319 (1975); A. A. Zvyagin and V. M. Tsukernik, Fiz. Tverd. Tela 30, 2857 (1988) [Sov. Phys. Solid State 30, 1649 (1988)]; A. Dutta, G. Aeppli, B. K. Chakrabarti, U. Divakaran, T. F. Rosenbaum, and D. Sen, Quantum Phase Transitions in Transverse Field Spin Models: From Statistical Physics to Quantum Information (Cambridge University Press, New Delhi, 2015).

[24] A. Kitaev, Ann. Phys. (NY) 321, 2 (2006).

[25] X.-Y. Feng, G.-M. Zhang, and T. Xiang, Phys. Rev. Lett. 98, 087204 (2007); H.-D. Chen and J. Hu, Phys. Rev. B 76, 193101 (2007); H.-D. Chen and Z. Nussinov, J. Phys. A: Math. Theor. 41, 075001 (2008).
[26] E. Fradkin, Field Theories of Condensed Matter Physics (Cambridge University Press, New York, 2013); N. Wu, Phys. Lett. A 376, 3530 (2012); M. Ezawa, Y. Tanaka, and N. Nagaosa, Sci. Rep. 3, 2790 (2013); Y. Niu, S. B. Chung, C.-H. Hsu, I. Mandal, S. Raghu, and S. Chakravarty, Phys. Rev. B 85, 035110 (2012).

[27] M. den Nijs and K. Rommelse, Phys. Rev. B 40, 4709 (1989); T. Kennedy and H. Tasaki, ibid. 45, 304 (1992); M. Kohmoto and H. Tasaki, ibid. 46, 3486 (1992); M. Oshikawa, J. Phys.: Condens. Matter 4, 7469 (1992).

[28] See, e.g., H. Yao and S. A. Kivelson, Phys. Rev. Lett. 99, 247203 (2007); S. Yang, D. L. Zhou, and C. P. Sun, Phys. Rev. B 76, 180404(R) (2007); C. Wu, D. Arovas, and H. H. Hung, ibid. 79, 134427 (2009); K. S. Tikhonov and M. V. Feigelman, Phys. Rev. Lett. 105, 067207 (2010); G. Kells, J. Kailasvouri, J. K. Slingerland, and J. Vala, New J. Phys. 13, 095014 (2011); M. Barkeshli, H.-C. Jiang, R. Thomale, and X.-L. Qi, Phys. Rev. Lett. 114, 026401 (2015); J.-J. Miao, H.-K. Jin, F. Wang, F.-C. Zhang, and Y. Zhou, Phys. Rev. B 99, 155105 (2019).

[29] Y. Aharonov and D. Bohm, Phys. Rev. 115, 485 (1959); Y. Aharonov and A. Casher, Phys. Rev. Lett. 53, 319 (1984).

[30] For the review, see, e.g., A. A. Zvyagin and I. V. Krive, Fiz. Nizk. Temp. 21, 687 (1995) [Low Temp. Phys. 21, 533 (1995).

[31] W. M. H. Natori, R. Moessner, and J. Knolle, Phys. Rev. B 100, 144403 (2019). 